\title{
The genetic bases of divergence in desiccation and starvation resistance among tropical and temperate populations of Drosophila melanogaster
}

\author{
W. J. KENNINGTON*, A. S. GILCHRIST, D. B. GOLDSTEIN \& L. PARTRIDGE \\ Department of Biology, Galton Laboratory, University College London, 4 Stephenson Way, London, NW1 2HE, U.K.
}

\begin{abstract}
Desiccation and starvation resistance are two stress-related traits which vary geographically with climate in Drosophila melanogaster. To investigate the contribution of epistasis to population divergence for these traits, we crossed tropical and temperate populations from two different geographical regions to produce $F_{1}, F_{2}$ and first backcross generations. Line-cross analysis of generation means revealed that genetic bases of divergence for both traits were complex and remarkably similar in a number of respects. Strong additive and dominance effects were present in most of the models, whereas epistatic and maternal effects were less common. The presence of epistatic effects in approximately half of the models presented in this study is consistent with line-cross studies of diverged traits in other animals, and does not support the view that epistasis is the predominant means by which populations diverge. In addition, evidence of maternal effects in both traits adds to a growing body of recent evidence that suggests maternal contributions to population differentiation are more widespread than previously thought. This finding undermines the accuracy of studies inferring epistasis directly from the magnitude of $F_{2}$ breakdown. More line-cross analysis studies of naturally diverged populations that take into account maternal effects will shed further light on the true incidence of epistasis and its importance in the evolutionary process.
\end{abstract}

Keywords: adaptive landscapes, epistasis, quantitative genetics, shifting-balance theory, stressresistance.

\section{Introduction}

The frequency with which epistasis or gene interactions contribute to genetic differentiation of natural populations continues to be a topic of considerable interest in evolutionary biology (e.g. Armbruster et al., 1997; Starmer et al., 1998; Gilchrist \& Partridge, 1999; Fenster \& Galloway, 2000). Epistatic contributions to population divergence can play an important role in the maintenance of genetic variation and in conservation genetics (see Fenster \& Galloway, 2000). However, the main interest in evolutionary biology is that epistasis may create adaptive peaks and valleys which can prevent deterministic evolution (see Whitlock et al., 1995; Fenster et al., 1997). If epistatic contributions to population divergence are common, then adaptive landscapes should be rugged, and peak shift models, such as Wright's shifting balance theory, are needed to

*Correspondence. E-mail: w.kennington@ucl.ac.uk explain evolutionary change. By contrast, if epistatic effects are uncommon, and adaptive landscapes are smooth, these models are unnecessary.

Until recently, empirical work on this fundamental issue has been limited, partly because of the absence of reliable, quantitative genetic approaches for quantifying epistasis (Whitlock et al., 1995; Fenster et al., 1997). One of the most statistically powerful methods is the hybrid breakdown approach. Here, divergent populations are crossed, and the offspring of resulting generations are measured to test the effect of the physical disruption of putative co-adapted gene complexes by recombination. In the simplest approach, epistatic contributions are estimated directly from the magnitude of reduced fitness in the $F_{2}$ generation compared to the mid-parent and $F_{1}$ hybrid generations. Line-cross or generation means analysis takes this approach a step further by comparing observed means of a variety of hybrid and backcross generations with expected means derived from genetic models that can account for 
additive, dominance, epistatic and maternal effects (see Mather \& Jinks, 1982; Kearsey \& Pooni, 1996; Lynch \& Walsh, 1998).

Results from hybrid breakdown studies to date suggest that epistatic contributions to the genetic differentiation observed between natural populations are the rule rather than the exception (for examples see Whitlock et al., 1995; Gilchrist \& Partridge, 1999). However, in many of these studies, epistatic parameters were not clearly separated from all other contributions by linecross analyses, making the actual incidence of epistasis in naturally diverged populations unclear. Further, Gilchrist \& Partridge (1999) have shown that a common assumption in many hybrid breakdown studies, that maternal effects are absent, can lead to the erroneous detection of epistasis. For example, in their study of three parallel clines in body size in Drosophila melanogaster, Gilchrist \& Partridge (1999) found significant maternal effects contributing to wing size divergence in most, but not all clines and, on at least four occasions, reduced size in the $F_{2}$ generation was exclusively due to these effects.

In Drosophila, desiccation and starvation resistance are two stress-resistant traits for which there is ample evidence for genetic differentiation among natural populations (see Hoffmann \& Harshman, 1999). Resistance to desiccation tends to be higher in populations from temperate areas compared to tropical ones, whereas the reverse pattern is evident for starvation resistance. Both traits therefore provide ideal opportunities to measure non-additive contributions to natural divergences, and hence shed further light on the ubiquity of epistasis. Here, we use line-cross analysis to dissect the genetic bases of divergence in these traits using tropical and temperate populations of D. melanogaster from two different geographical regions.

\section{Materials and methods}

\section{Fly populations}

Fly populations were selected from tropical and temperate localities in two geographical regions in the southern hemisphere, Australasia (a geographical region that includes Australia and Papua New Guinea) and South America. The Australasian populations were a temperate population from Cygnet in southern Australia $\left(43.08^{\circ} \mathrm{S}, 147.24^{\circ} \mathrm{E}\right)$, and a tropical population from Madang in Papua New Guinea $\left(5.32^{\circ} \mathrm{S}, 145.70^{\circ} \mathrm{E}\right)$. The Cygnet population was collected in January 1997, and the Madang population was collected in August 1998. The South American populations were a temperate population from Porto Montt in southern Chile $\left(41.50^{\circ} \mathrm{S}, 72.83^{\circ} \mathrm{W}\right)$ and a tropical population from Guayaquil in Ecuador $\left(2.22^{\circ} \mathrm{S}, 79.90^{\circ} \mathrm{W}\right)$. These popu- lations were collected during February and March 1995 and were provided by Dr Jan Van't Land. All stocks were maintained as bottle stocks at $25^{\circ} \mathrm{C}$ on a $12: 12 \mathrm{~h}$ light : dark cycle on a standard cornmeal/yeast/sugar medium.

With the exception of the Madang population, all the flies were collected en masse. For the Madang population, the flies were originally collected as isofemale lines. After four months in the laboratory, a population was established for this locality by bulking samples of flies from 20 isofemale lines.

\section{Crosses}

For each geographical region, the temperate and tropical populations were crossed to produce six basic generations, the two parental populations $\mathrm{P}_{1}$ and $\mathrm{P}_{2}$, their $F_{1}$ and $F_{2}$ generations, and the two backcross generations, $B_{1}$ and $B_{2}$ (i.e. $F_{1} \times P_{1}$ and $F_{1} \times P_{2}$ ). Reciprocals for all crosses were also established, and were denoted as $F_{1} R, F_{2} R, B_{1} R$ and $B_{2} R$. In addition, for each $F_{2}$ and backcross generation, separate generations were raised in which the $F_{1}$ parent differed reciprocally [e.g. $\left(\mathrm{P}_{1} \times \mathrm{P}_{2}\right) \times \mathrm{P}_{1}$ and $\left(\mathrm{P}_{2} \times \mathrm{P}_{1}\right) \times \mathrm{P}_{1}$ ]. These additional crosses were designated $\mathrm{F}_{2} \mathrm{a}, \mathrm{F}_{2} \mathrm{~b}$, $\mathrm{F}_{2} \mathrm{Ra}$ and $\mathrm{F}_{2} \mathrm{Rb}$ for the $\mathrm{F}_{2}$ generations, and $\mathrm{B}_{1} \mathrm{a}, \mathrm{B}_{1} \mathrm{Ra}$, etc. for the backcross generations. In total, 16 distinct generations were raised from each geographical region. All crosses were set up with at least 25 virgin individuals of each sex as parents. Progeny derived from these crosses were reared at a standard larval density (50 larvae per vial) at $25^{\circ} \mathrm{C}$ by allowing parents to oviposit on a grape juice/agar medium and then transferring first instar larvae to new vials containing unyeasted standard food medium. Ten replicate vials were established for each cross. After nine days, virgin flies of each sex were collected from each vial over a two-day period and transferred to new vials containing unyeasted standard food medium. Flies from replicate vials and each sex were kept unmated in separate vials at $25^{\circ} \mathrm{C}$ until used in the experimental assays. Just prior to being measured, flies in each replicate vial were allocated randomly and equally to two groups, so that half the flies could be measured for desiccation resistance and the other half for starvation resistance. Crosses from the two geographical regions were reared and measured for resistance at different times in November 1999.

\section{Desiccation measurement}

Desiccation resistance was measured on 5-6-day-old virgin flies, and up to 14 flies (mean $=7.4$ ) of each sex were measured for each of the 10 replicate vials of each cross. To measure resistance, flies from each vial were 
transferred to a new vial containing a disc of dry filter paper and covered with muslin gauze cloth secured with an elastic band. Desiccation vials were kept at $25^{\circ} \mathrm{C}$ under constant light and were observed for the number of dead flies seven hours after the flies were originally transferred, and then at half-hourly intervals until all the flies had died. Following Robinson et al. (2000), death was deemed to have occurred when a fly showed no sign of movement, even after the vial was shaken lightly. Because all the flies were transferred to the desiccation vials at different times, the time of transfer was also recorded and survival times adjusted accordingly.

In contrast to the established technique of using a desiccator containing silica gel to create a low humidity environment to measure desiccation resistance, survival times in this experiment were measured at room humidity (30-40\% RH). This approach has the advantage of imposing a biologically more realistic stress on the flies, and, because survival times are longer than those observed with a desiccator, it also produces a greater number of measurement intervals during the experiment. A preliminary experiment confirmed that the same differences in desiccation resistance between parental populations were evident using the two methods.

\section{Starvation measurement}

Starvation resistance was measured on 4-5-day-old virgin flies and, as with desiccation resistance, up to 14 flies (mean $=7.4$ ) of each sex were measured for each of the 10 replicate vials of each cross. To measure starvation resistance, flies from each vial were transferred to a new vial containing $7 \mathrm{~mL}$ of $1 \%$ agar and plugged with cotton wool in order to prevent desiccation. Starvation vials were kept at $25^{\circ} \mathrm{C}$ under constant light, and were observed for the number of dead flies $40 \mathrm{~h}$ after the flies were originally transferred, and then at six-hourly intervals until all the flies had died.

\section{Analysis of differences between parental populations}

To determine if significant differences in desiccation and starvation resistance existed among parental populations within each geographical region, nested analysis of variance (with vial nested within population) were performed on individual survival times using the JMP 3.2.2 statistical package (SAS, 1997). Each sex was analysed independently. In all cases, analyses were performed on logarithmic transformations of survival time, because this tended to improve fits to the normal distribution. On no occasion did data transformations change the level of significance of a result.

\section{Analysis of line-crosses}

All line-cross analyses were performed separately on each sex for each geographical region on logarithmic transformations of survival times. Generation means were analysed using methods described in Mather \& Jinks (1982), and are outlined here in brief (for a more detailed description of the methods used see Gilchrist \& Partridge, 1999). First, the observed means were used to estimate the parameters of a model consisting only of an overall mean, $m$, and the composite additive $[a]$ and dominance $[d]$ effects (symbols used follow those adopted by Kearsey \& Pooni, 1996). These estimated parameters were used, in turn, to calculate expected generation means. Goodness of fit between observed and expected generation means was tested using $\chi^{2}$, and in instances where a simple additive-dominance model was insufficient to explain the data, further parameters were added to the model. The additional parameters were added in the following order: first, composite digenic effects (additive $\times$ additive $[a a]$, additive $\times$ dominance $[a d]$ and dominance $\times$ dominance $[d d]$ ); second, parameters to account for maternal effects; and finally, a parameter to account for Y-linked effects $[Y]$. The maternal effect parameters were: additive $[a]_{\mathrm{m}}$ and dominance $[d]_{\mathrm{m}}$ maternal effects, interactions between additive and dominance effects in the progeny and maternal effects $\left(a . a_{\mathrm{m}}, a \cdot d_{\mathrm{m}}, d \cdot a_{\mathrm{m}}\right.$ and $\left.d . d_{\mathrm{m}}\right)$, and cytoplasmic effects [c], which account for mitochondrial genetic effects, symbionts and infectious agents.

The parameter coefficients used to test the models are given in Table 1 . From Table 1 it is evident that $[a]_{\mathrm{m}}$ effects are only apparent in generations where the mother is from either of the parental populations $\left(\mathrm{P}_{1}\right.$ or $\left.\mathrm{P}_{2}\right)$, and $[d]_{\mathrm{m}}$ effects are only apparent in generations where the mother is an $F_{1}$ hybrid. By contrast, cytoplasmic effects $[c]$ depend simply on the line of descent of cytoplasm through the maternal line. Because 16 generations were used, models containing all 14 parameters could be tested for significance. In all cases, models were constructed to contain the least number of parameters necessary to explain the observed generation means, and additional parameters were only added only if they significantly improved the fit of the model.

Parameter coefficients used in our analysis followed Mather \& Jinks (1982) in being based on an $F-\infty$ metric, as opposed to the $F_{2}$ metric used by Lynch \& Walsh (1998). Because these metrics use a different reference point $m$, for defining expected generation means, the expectations can look rather different. However, Gilchrist \& Partridge (1999) found that the two systems produce very similar parameter estimates, and for this reason we deemed it unnecessary to use both. 
Table 1 Parameter coefficients used in models for estimating composite genetic effects. See Kearsey \& Pooni (1996) for details on the assignment of parameter coefficients to each cross

\begin{tabular}{|c|c|c|c|c|c|c|c|c|c|c|c|c|c|c|c|}
\hline & $\begin{array}{l}\text { Mather \& Jinks notation: } \\
\text { Edinburgh/Iowa notation: }\end{array}$ & $\begin{array}{l}m \\
m\end{array}$ & $\begin{array}{l}{[d]} \\
{[a]} \\
\end{array}$ & $\begin{array}{l}{[h]} \\
{[d]} \\
\end{array}$ & $\begin{array}{c}{[i]} \\
{[a a]}\end{array}$ & $\begin{array}{c}{[j]} \\
{[a d]}\end{array}$ & $\begin{array}{c}{[l]} \\
{[d d]}\end{array}$ & $\begin{array}{l}{[d] m} \\
{[a] m}\end{array}$ & $\begin{array}{l}{[h] m} \\
{[d] m}\end{array}$ & $\begin{array}{l}\text { d. } d_{\mathrm{m}} \\
\text { a. } a_{\mathrm{m}}\end{array}$ & $\begin{array}{l}d . h_{\mathrm{m}} \\
a \cdot d_{\mathrm{m}}\end{array}$ & $\begin{array}{l}h \cdot d_{\mathrm{m}} \\
\text { d. } a_{\mathrm{m}}\end{array}$ & $\begin{array}{l}h . h_{\mathrm{m}} \\
d . d_{\mathrm{m}}\end{array}$ & {$[c]$} & {$[Y]$} \\
\hline $\mathrm{P}_{1}$ & e.g. Madang & 1 & 1 & 0 & 1 & 0 & 0 & 1 & 0 & 1 & 0 & 0 & 0 & 1 & 1 \\
\hline $\mathrm{P}_{2}$ & e.g. Cygnet & 1 & -1 & 0 & 1 & 0 & 0 & -1 & 0 & 1 & 0 & 0 & 0 & -1 & -1 \\
\hline$F_{1}$ & $\mathrm{P}_{1} \times \mathrm{P}_{2}$ & 1 & 0 & 1 & 0 & 0 & 1 & 1 & 0 & 0 & 0 & 1 & 0 & 1 & -1 \\
\hline$F_{1} R$ & $\mathrm{P}_{2} \times \mathrm{P}_{1}$ & 1 & 0 & 1 & 0 & 0 & 1 & -1 & 0 & 0 & 0 & -1 & 0 & -1 & 1 \\
\hline $\mathrm{F}_{2} \mathrm{a}$ & $\left(\mathrm{P}_{1} \times \mathrm{P}_{2}\right) \times\left(\mathrm{P}_{1} \times \mathrm{P}_{2}\right)$ & 1 & 0 & 0.5 & 0 & 0 & 0.25 & 0 & 1 & 0 & 0 & 0 & 0.5 & 1 & -1 \\
\hline $\mathrm{F}_{2} \mathrm{~b}$ & $\left(\mathrm{P}_{1} \times \mathrm{P}_{2}\right) \times\left(\mathrm{P}_{2} \times \mathrm{P}_{1}\right)$ & 1 & 0 & 0.5 & 0 & 0 & 0.25 & 0 & 1 & 0 & 0 & 0 & 0.5 & 1 & 1 \\
\hline $\mathrm{F}_{2} \mathrm{Ra}$ & $\left(\mathrm{P}_{2} \times \mathrm{P}_{1}\right) \times\left(\mathrm{P}_{1} \times \mathrm{P}_{2}\right)$ & 1 & 0 & 0.5 & 0 & 0 & 0.25 & 0 & 1 & 0 & 0 & 0 & 0.5 & -1 & -1 \\
\hline $\mathrm{F}_{2} \mathrm{Rb}$ & $\left(\mathrm{P}_{2} \times \mathrm{P}_{1}\right) \times\left(\mathrm{P}_{2} \times \mathrm{P}_{1}\right)$ & 1 & 0 & 0.5 & 0 & 0 & 0.25 & 0 & 1 & 0 & 0 & 0 & 0.5 & -1 & 1 \\
\hline $\mathrm{B}_{1} \mathrm{a}$ & $\mathrm{P}_{1} \times\left(\mathrm{P}_{1} \times \mathrm{P}_{2}\right)$ & 1 & 0.5 & 0.5 & 0.25 & 0.25 & 0.25 & 1 & 0 & 0.5 & 0 & 0.5 & 0 & 1 & $\begin{array}{llll}-1 & & & \end{array}$ \\
\hline $\mathrm{B}_{1} \mathrm{~b}$ & $\mathrm{P}_{1} \times\left(\mathrm{P}_{2} \times \mathrm{P}_{1}\right)$ & 1 & 0.5 & 0.5 & 0.25 & 0.25 & 0.25 & 1 & 0 & 0.5 & 0 & 0.5 & 0 & 1 & 1 \\
\hline $\mathrm{B}_{1} \mathrm{Ra}$ & $\left(\mathrm{P}_{1} \times \mathrm{P}_{2}\right) \times \mathrm{P}_{1}$ & 1 & 0.5 & 0.5 & 0.25 & 0.25 & 0.25 & 0 & 1 & 0 & 0.5 & 0 & 0.5 & 1 & 1 \\
\hline $\mathrm{B}_{1} \mathrm{Rb}$ & $\left(\mathrm{P}_{2} \times \mathrm{P}_{1}\right) \times \mathrm{P}_{1}$ & 1 & 0.5 & 0.5 & 0.25 & 0.25 & 0.25 & 0 & 1 & 0 & 0.5 & 0 & 0.5 & -1 & 1 \\
\hline $\mathrm{B}_{2} \mathrm{a}$ & $\mathrm{P}_{2} \times\left(\mathrm{P}_{1} \times \mathrm{P}_{2}\right)$ & 1 & -0.5 & 0.5 & 0.25 & -0.25 & 0.25 & -1 & 0 & 0.5 & 0 & -0.5 & 0 & -1 & -1 \\
\hline $\mathrm{B}_{2} \mathrm{~b}$ & $\mathrm{P}_{2} \times\left(\mathrm{P}_{2} \times \mathrm{P}_{1}\right)$ & 1 & -0.5 & 0.5 & 0.25 & -0.25 & 0.25 & -1 & 0 & 0.5 & 0 & -0.5 & 0 & -1 & 1 \\
\hline $\mathrm{B}_{2} \mathrm{Ra}$ & $\left(\mathrm{P}_{1} \times \mathrm{P}_{2}\right) \times \mathrm{P}_{2}$ & 1 & -0.5 & 0.5 & 0.25 & -0.25 & 0.25 & 0 & 1 & 0 & -0.5 & 0 & 0.5 & 1 & -1 \\
\hline $\mathrm{B}_{2} \mathrm{Rb}$ & $\left(\mathrm{P}_{2} \times \mathrm{P}_{1}\right) \times \mathrm{P}_{2}$ & 1 & -0.5 & 0.5 & 0.25 & -0.25 & 0.25 & 0 & 1 & 0 & -0.5 & 0 & 0.5 & -1 & -1 \\
\hline
\end{tabular}

Model parameters, their errors and $\chi^{2}$ values were estimated using weighted least-squares methods, expanded to incorporate vial variances. For a detailed description of these methods and the formula used see Gilchrist \& Partridge (1999). Because experiments were not always fully balanced, which could, in theory, add sources of error to the analyses, grid searches of multidimensional plots of likelihood surfaces were also used to estimate parameters that were found to significantly improve the model (see Lynch \& Walsh, 1998). In all cases, estimates of model parameters from the two methods were found to be highly similar (within one standard error), and for this reason, estimates from the grid searches are not reported here.

\section{Results}

\section{Differences between parental populations}

Desiccation resistance. For all comparisons except the Australasian females, nested analysis of variance revealed significant differences in survival times among parental populations $(P<0.001$ in all significant cases $)$. For the South American populations, mean survival times in both sexes were greater in flies from the temperate locality (29.0 vs. $21.4 \mathrm{~h}$ and $16.2 \mathrm{vs.} 13.0 \mathrm{~h}$ for females and males, respectively), whereas for the Australasian populations, flies from the tropical locality had the greater mean survival times ( 22.9 vs. $21.2 \mathrm{~h}$ and 19.9 vs. $12.3 \mathrm{~h}$ for females and males, respectively). The largest difference among means was in Australasian males $(7.6 \mathrm{~h})$, and the smallest difference was in
Australasian females $(1.7 \mathrm{~h})$. In all populations, mean survival time was significantly greater in females than in males $(P<0.001$ in all cases).

In all cases, shorter survival times of all flies in the desiccation experiment, compared to the minimum survival time in the starvation experiment for each sex, confirmed that desiccation, rather than starvation, was the cause of mortality.

Starvation resistance. For all comparisons, nested analysis of variance revealed significant differences in survival times among parental populations $(P<0.001$ in all cases except Australasian females, where $P=0.04$ ). As found in the desiccation experiment, mean survival times of both sexes were greater in flies from the temperate locality for the South American populations (135.7 vs. $99.8 \mathrm{~h}$ and 113.0 vs. $68.5 \mathrm{~h}$ for females and males, respectively), and greater in flies from the tropical locality for the Australasian populations (130.6 vs. $111.7 \mathrm{~h}$ and $91.5 \mathrm{vs} .74 .6 \mathrm{~h}$ for females and males, respectively). Similarly, mean survival time was significantly greater in females in all populations $(P<0.001$ in all cases). The largest difference between parental means was in South American males (44.5 h), and the smallest difference was in Australasian females $(13.5 \mathrm{~h})$. The difference between South American populations was consistent with those reported by Robinson et al. (2000).

\section{Line-cross analyses}

Desiccation resistance. For the desiccation experiment, the mean survival times of both sexes in all 16 

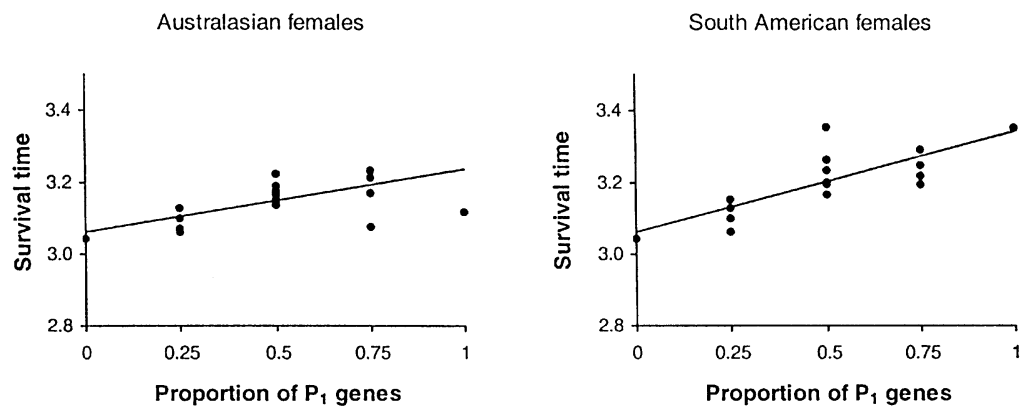

Fig. 1 Mean survival times of Drosophila melanogaster in $\ln$ (hours) for the desiccation resistance experiment as a function of the proportion of genes derived from $\mathrm{P}_{1}$, the most resistant parent, in each cross. The solid line is the weighted least-squares estimate of a simple additive model. For clarity, standard error bars are not shown. In no instance did the standard error exceed $3 \times$ the symbol diameter.

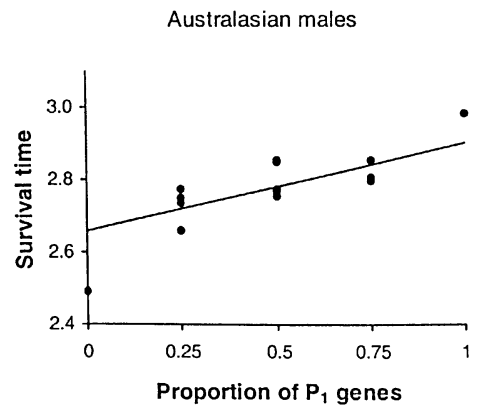

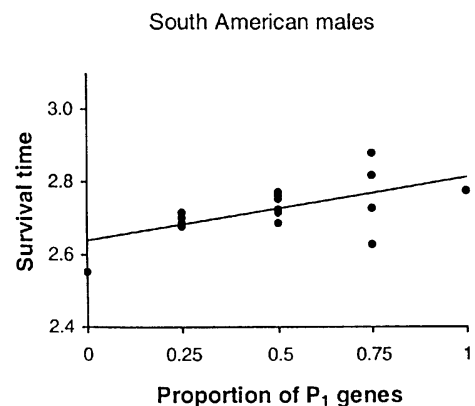

generations from both geographical regions are shown in Fig. 1. In each graph, the regression line represents the weighted least-squares estimate of a simple genetic model containing overall mean and additive effects only. In no case was the simple additive model sufficient to describe the observed means (minimum $\chi^{2}=41.42$, $P<0.001$, for the Australasian males). Also worthy of note is that the observed means of Australasian females for all $F_{1}$ and $F_{2}$ generations were greater than those exhibited by either parent. This pattern is known as transgressive segregation and is most likely to be due to complementary gene action or overdominance (see Rieseberg et al., 2000).

Best-fitting models explaining the observed generation means are given in Table 2. A number of general observations are evident. First, a simple additivedominance model did not adequately describe the observed means in any set of generations, and it was necessary to test additional parameters to improve the goodness of fit. The closest fit between the simple additive-dominance model and observed means was for the Australasian females $\left(\chi^{2}=31.95, P<0.01\right)$. In this case, no additional parameters could be found to significantly improve the fit of the model, although $[a d]$ effects were only marginally nonsignificant. When digenic epistatic and maternal effects were included in the remaining models, both sets of male generation means were adequately described (i.e. the $\chi^{2}$ for both models were nonsignificant). However, in both female data sets, no models could be found to adequately describe the observed means, suggesting that linkage, and/or higher order interactions (e.g. trigenic interactions) are necessary to fully explain these means. This is not surprising considering the low recombination indices of D. melanogaster (see Lynch \& Walsh, 1998), but contrasts with models describing morphological divergence in $D$. melanogaster that are adequately explained without linkage or higher order interactions (see Gilchrist \& Partridge, 1999).

Second, digenic epistatic interactions were present in males from both geographical regions, but absent in females. However, in the Australasian females [ad] effects were only marginally nonsignificant, and in the South American females, significant $[a a]$ and $[d d]$ effects were found, but since they were not independently significant, and were opposite in sign, we did not include them in the final model. Significant $[a d]$ effects were present in two models (South American males and Australasian males) and were negative or opposite from additive effects in both cases. Significant $[a a]$ effects were present in only one model (South American males) and were positive in sign.

Finally, additive maternal effects were present in both sexes in South American populations, but were notably absent in the Australasian populations. In the South American crosses, additive maternal effects were positive in females and negative in males. Dominance maternal effects were found in only one model (South American males). The only instance of an $a \cdot d_{\mathrm{m}}$ effect, or any other interaction between maternal and progeny effects, was 


\begin{tabular}{|c|c|c|c|}
\hline \multirow[b]{2}{*}{ Sex } & \multirow[b]{2}{*}{ Parameter } & \multicolumn{2}{|c|}{ Region } \\
\hline & & Australasia & South America \\
\hline \multirow[t]{9}{*}{ Females } & $m$ & $3.102 \pm 0.014 * * *$ & $3.131 \pm 0.014 * * *$ \\
\hline & {$[a]$} & $0.077 \pm 0.012 * * *$ & $0.071 \pm 0.016^{* * *}$ \\
\hline & {$[d]$} & $0.089 \pm 0.024 * * *$ & $0.124 \pm 0.021 * * *$ \\
\hline & {$[a a]$} & & \\
\hline & {$[a d]$} & & \\
\hline & {$[d d]$} & & \\
\hline & {$[a]_{\mathrm{m}}$} & & $0.054 \pm 0.009 * * *$ \\
\hline & {$[d]_{\mathrm{m}}$} & & \\
\hline & $\chi^{2}$ & $32.11^{* *}$ & $58.39 * * *$ \\
\hline \multirow[t]{10}{*}{ Males } & $m$ & $2.731 \pm 0.021 * * *$ & $2.571 \pm 0.037 * * *$ \\
\hline & {$[a]$} & $0.247 \pm 0.028 * * *$ & $0.159 \pm 0.024 * * *$ \\
\hline & {$[d]$} & $0.085 \pm 0.038^{*}$ & $0.160 \pm 0.047 * * *$ \\
\hline & {$[a a]$} & & $0.096 \pm 0.043^{*}$ \\
\hline & {$[a d]$} & $-0.351 \pm 0.066^{* * *}$ & $-0.256 \pm 0.060 * * *$ \\
\hline & {$[d d]$} & & \\
\hline & {$[a]_{\mathrm{m}}$} & & $-0.046 \pm 0.015^{* *}$ \\
\hline & {$[d]_{\mathrm{m}}$} & & $0.095 \pm 0.015 * * *$ \\
\hline & a. $d_{\mathrm{m}}$ & & $0.153 \pm 0.043 * * *$ \\
\hline & $\chi^{2}$ & $15.47 \mathrm{NS}$ & 15.37 NS \\
\hline
\end{tabular}

NS, not significant.

${ }^{*} P<0.05 ; * * P<0.01 ; * * * P<0.001$.

Australasian females

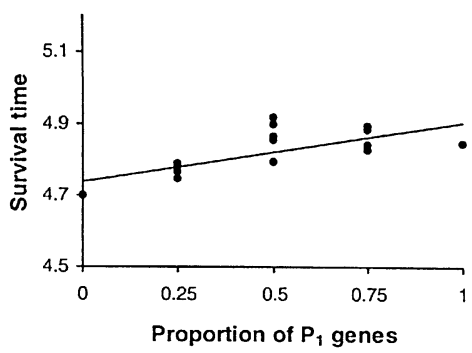

Australasian males

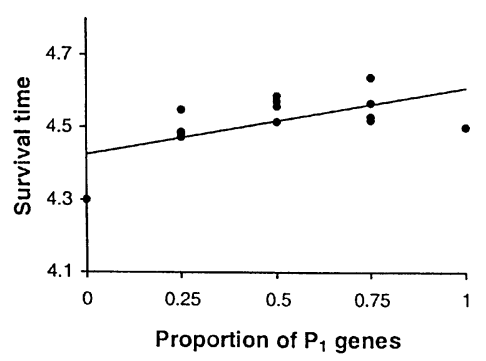

South American females

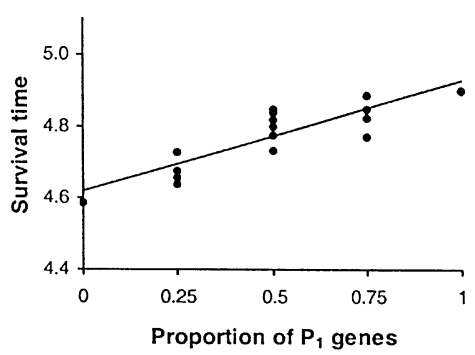

South American males

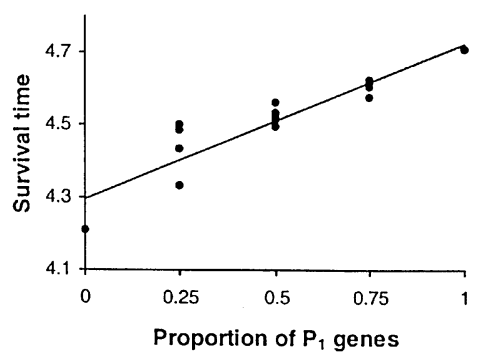

Table 2 Estimates of composite genetic effects underlying divergence in desiccation resistance between the tropical and temperate populations of Drosophila melanogaster from the two geographical regions. The $\chi^{2}$-values were calculated using models that include only the parameters whose values are shown. The number of asterisks indicates the significance of the improved fit when the parameter was added
Fig. 2 Mean survival times of Drosophila melanogaster in $\ln$ (hours) for the starvation resistance experiment as a function of the proportion of genes derived from $\mathrm{P}_{1}$, the most resistant parent, in each cross. The solid line is the weighted least-squares estimate of a simple additive model. For clarity, standard error bars are not shown. In no instance did the standard error exceed $2.5 \times$ the symbol diameter. also present in this model. In both cases, the effects were positive in sign. There were no significant cytoplasmic or $Y$ effects in any model.

Starvation resistance. For the starvation experiment, the mean survival times of both sexes in all 16 generations from both geographical regions are shown in Fig. 2. As before, the regression line in each graph is the weighted least-squares estimate of a simple genetic model containing overall mean and additive effects only. Similarly, in no case was the simple additive model sufficient to describe the observed means (minimum $\chi^{2}=33.18$, 
Table 3 Estimates of composite genetic effects underlying divergence in starvation resistance between the tropical and temperate populations of Drosophila melanogaster from the two geographical regions. Explanation of entries as for Table 2

\begin{tabular}{|c|c|c|c|}
\hline \multirow[b]{2}{*}{ Sex } & \multirow[b]{2}{*}{ Parameter } & \multicolumn{2}{|c|}{ Region } \\
\hline & & Australasia & South America \\
\hline \multirow[t]{9}{*}{ Females } & $m$ & $4.760 \pm 0.019 * * *$ & $4.721 \pm 0.016^{* * *}$ \\
\hline & [a] & $0.137 \pm 0.018 * * *$ & $0.125 \pm 0.015^{* * *}$ \\
\hline & {$[d]$} & $0.106 \pm 0.035^{* *}$ & \\
\hline & {$[a a]$} & & \\
\hline & {$[a d]$} & & \\
\hline & {$[d d]$} & & \\
\hline & {$[a]_{\mathrm{m}}$} & $-0.042 \pm 0.013 * *$ & $0.029 \pm 0.010^{* *}$ \\
\hline & {$[d]_{\mathrm{m}}$} & $0.037 \pm 0.012 * *$ & $0.058 \pm 0.011 * * *$ \\
\hline & $\chi^{2}$ & $26.33 * *$ & $31.25 * *$ \\
\hline \multirow[t]{9}{*}{ Males } & $m$ & $4.399 \pm 0.013 * * *$ & $4.460 \pm 0.015^{* * *}$ \\
\hline & {$[a]$} & $0.100 \pm 0.013 * * *$ & $0.268 \pm 0.019^{* * *}$ \\
\hline & $\begin{array}{l}{[d]} \\
{[a a]}\end{array}$ & $0.347 \pm 0.051 * * *$ & $0.055 \pm 0.021 * *$ \\
\hline & {$[a d]$} & $-0.113 \pm 0.044 * *$ & $-0.134 \pm 0.048^{* *}$ \\
\hline & {$[d d]$} & $-0.166 \pm 0.048 * * *$ & \\
\hline & {$[a]_{\mathrm{m}}$} & & \\
\hline & {$[d]_{\mathrm{m}}$} & & $0.043 \pm 0.012 * * *$ \\
\hline & $Y$ & & $-0.016 \pm 0.006^{* *}$ \\
\hline & $\chi^{2}$ & $17.78 \mathrm{NS}$ & $33.62 * * *$ \\
\hline
\end{tabular}

NS, not significant.

$* P<0.05 ; * * P<0.01 ; * * * P<0.001$
$P<0.01$, for the Australasian females). There was also evidence of transgressive segregation in the Australasian generations, but this time it was evident in both sexes. In males, all $F_{1}$ and $F_{2}$ generations had mean survival times greater than both parents, but in females one of the four $\mathrm{F}_{2}$ generations was not transgressive.

Models with the fewest parameters producing the best fit to the observed generation means are given in Table 3. As with the desiccation experiment, a number of observations can be made by comparing the different models. First, as before, a simple additive-dominance model did not adequately describe the observed means in any set of generations and additional parameters were necessary to improve the goodness of fit. Once again, the closest fit between the simple additive-dominance model and observed means was for the Australasian females $\left(\chi^{2}=22.47, \quad P<0.05\right)$. When digenic epistatic and maternal effects were included in the remaining models, only the Australasian female means were adequately described, suggesting that, for these models too, linkage and/or higher order interactions (e.g. trigenic interactions) may be having an effect.

Second, digenic epistatic interactions were again present in males from both geographical regions, but absent in both sets of female generations. It should be noted here, however, that if dominance effects were not included in the model for South American females, $[d d]$ effects could have been added. Significant $[a d]$ effects were present in only one model (South American males) and, as before, were opposite in sign to the additive effects. Likewise, significant $[d d]$ effects were present in only one model (Australasian males) and, consistent with the trend noted by Kearsey \& Pooni (1996), were opposite in sign to the $[d]$ effects. Significant $[a a]$ effects were not present in any model.

Finally, significant additive maternal effects were present in only two models (Australasian females and South American females), as were dominance maternal effects (both South American generations). For the South American generations, all maternal effects were positive, but in the one instance of maternal effects in the Australasian generations, they were negative in sign. A significant $a . d_{\mathrm{m}}$ effect was also present in an Australasian generation, and this too was negative. As in the desiccation experiment, there were no significant cytoplasmic effects in any model, but a significant negative $Y$ effect of small magnitude was present in South American males.

\section{Discussion}

Two striking observations on the differences between populations arising from this study are the positive associations between desiccation and starvation resistance, and the opposing patterns of divergence for each trait in different geographical regions. These results 
differ from studies on Drosophila that show clinal patterns of increased resistance with latitude for desiccation resistance and decreasing resistance with latitude for starvation resistance (e.g. Karan et al., 1998). However, they are consistent with studies showing deviations from clinal patterns for both desiccation (e.g. Parsons, 1980; Coyne et al., 1983; Da Lage et al., 1990; Blows \& Hoffmann, 1993; Hoffmann et al., in press) and starvation resistance (e.g. Robinson et al., 2000). In addition, a recent study by Hoffmann et al. (in press) using independent collections of D. melanogaster from tropical and temperate localities in Australia, has shown that tropical populations have significantly higher desiccation and starvation resistance than temperate populations, as found with the Australasian populations in this study. Positive associations between desiccation and starvation resistance have also been observed in D. melanogaster following artificial selection for increased resistance in either trait (e.g. Hoffmann \& Parsons, 1989, 1993; Harshman et al., 1999), and this suggests there may be common mechanisms underlying variation in these traits.

The line-cross analyses revealed a number of strong similarities among best-fitting models for both traits in both geographical regions. Foremost amongst these is the absence of any form of epistasis in females, and the low incidence of additive $\times$ additive interactions overall. Of the eight models presented in this study ( 2 traits $\times 2$ regions $\times 2$ sexes), only one had a significant additive $x$ additive effect, whereas four had significant additive $x$ dominance interactions and one a significant dominance $\times$ dominance interaction. Some form of digenic epistasis was present in four of the best-fitting models in this study ( $57 \%$ of the models with significantly diverged populations), and this is consistent with the incidence of epistasis in line-cross analysis studies of other naturally diverged animal populations (Table 4). The lack of epistasis in almost half of these models is not consistent with the view that epistatic selection is the predominant means by which populations diverge (e.g. Wright, 1931, 1977; Wade \& Goodnight, 1991), as it is unlikely that gene interactions would disappear after prior involvement in the process of evolutionary divergence.

Another similarity among the models reported in this study is the common occurrence of maternal effects. Five $(62 \%)$ of the models in this study have significant contributions by either additive or dominance maternal effects. Maternal effects have been found previously for desiccation resistance in interspecific Drosophila crosses (Hercus \& Hoffmann, 1999), and were present in over half of the genetic models accounting for population differentiation in wing size (Table 4 ). The possibility of maternal effects being widespread has important implications for hybrid breakdown studies, because unaccounted maternal effects can contribute to highly misleading conclusions about the importance of epistasis. If, as suggested from this and recent studies, maternal effects are more common than first thought, results from hybrid breakdown studies should be re-appraised; this may influence the current consensus suggesting that epistasis is common between populations that have differentiated under selection.

A final point of interest arising from comparisons of genetic models in this study is the high incidence of positive dominance effects. Almost all of the models in this study had significant additive and dominance contributions. These results compare well with McKenzie \& Parsons (1974), who consistently found significant additive and dominance effects underlying differences in desiccation resistance among lines within populations of D. melanogaster, but are contrasting to the absence of dominance in lines selected for increased desiccation resistance (Hoffmann \& Parsons, 1989). Since directional selection is expected to produce more dominance

Table 4 Summary of line-cross analyses of diverged animal populations. Numbers outside parentheses indicate the number of best-fit models in which the genetic parameter was significant. Numbers inside parentheses indicate the total number of models presented. NA indicates a parameter that was not assessed. NA* indicates the parameter was not assessed, but preliminary comparisons of reciprocal crosses had shown significant effects in only a few generations in the analysis

\begin{tabular}{lllllll}
\hline & \multicolumn{3}{c}{ Genetic parameter } & & & \\
\cline { 2 - 4 } Species & \multicolumn{1}{c}{ Trait } & {$[a]$} & {$[d]$} & Epistasis & Maternal & \multicolumn{1}{c}{ Reference } \\
\hline Drosophila hibisci & Ovariole number & $1(1)$ & $0(1)$ & $1(1)$ & $0(1)$ & Starmer et al. (1998) \\
D. melanogaster & $\begin{array}{l}\text { Knockdown resistance } \\
\text { to ethanol fumes }\end{array}$ & $3(3)$ & $3(3)$ & $0(3)$ & NA & Cohan et al. (1989) \\
& & & & & \\
D. melanogaster & Wing area & $6(6)$ & $6(6)$ & $3(6)$ & $4(6)$ & Gilchrist \& Partridge (1999) \\
D. melanogaster & Desiccation resistance & $4(4)$ & $4(4)$ & $2(4)$ & $2(4)$ & This study \\
D. melanogaster & Starvation resistance & $4(4)$ & $3(4)$ & $2(4)$ & $3(4)$ & This study \\
Wyeomyia smithii & Photoperiodism & $2(2)$ & $1(2)$ & $2(2)$ & NA* & Hard et al. (1992) \\
\hline
\end{tabular}


than stabilizing selection (Mather, 1966), McKenzie \& Parsons (1974) suggested that directional selection was likely to have contributed to their result. Similarly, directional selection may also have contributed to the dominance of the most stress-resistant alleles in this study.

Our study has shown that the genetic bases of existing differences in desiccation and starvation resistance among temperate and tropical populations are complex and remarkably similar in a number of respects. Strong additive and dominance effects were present in most models, whereas significant epistatic and maternal effects were less common. However, it is possible that adaptation to laboratory culture has led to an underestimation of the true genetic effects underlying differentiation between natural populations. Nevertheless, the presence of epistasis in approximately half of the models presented in this study is consistent with line-cross analysis studies of diverged traits in other animals. These results do not provide support for the view that epistatic selection is the predominant means by which populations diverge. More line-cross analysis studies of naturally diverged populations that take into account maternal effects will shed further light on the true incidence of epistasis and allow its likely importance in the evolutionary process to be properly assessed.

\section{Acknowledgements}

We thank the Natural Environment Research Council for financial support. We are also grateful to Jon Bridle and two anonymous referees for helpful comments that improved this manuscript.

\section{References}

ARMBRUSTER, P., BRADSHAW, W. E. AND HOLZAPFEL, C. M. 1997. Evolution of the genetic architecture underlying fitness in the pitcher-plant mosquito, Wyeomyia smithii. Evolution, 51, 451-458.

BLOWS, M. W. AND HOFFMANN, A. A. 1993. The genetic of central and marginal populations of Drosophila serrata. I. Genetic variation for stress resistance and species borders. Evolution, 47, 1255-1270.

COHAN, F. M., HOFFMANN, A. A. AND GAYLEY, T. W. 1989. A test for the role of epistasis in divergence under uniform selection. Evolution, 43, 766-774.

COYNE, J. A., BUNDGAARD, J. AND PROUT, T. 1983. Geographic variation of tolerance to environmental stress in Drosophila pseudoobscura. Am. Nat., 122, 474-488.

DA LAGE, J. L., CAPY, P. AND DAVID, J. R. 1990. Starvation and desiccation in Drosophila melanogaster: differences between European, North African and Afrotropical populations. Génét. Sél. Évol., 22, 381-391.
FENSTER, C. B. AND GAlloway, L. F. 2000. Population differentiation in an annual legume: genetic architecture. Evolution, 54, 1157-1172.

FENSTER, C. B., GALlOWAY, L. F. AND CHAO, L. 1997. Epistasis and its consequences for the evolution of natural populations. Trends Ecol. Evol., 12, 282-286.

GILCHRIST, A. S. AND PARTRIDGE, L. 1999. A comparison of the genetic basis of wing size divergence in three parallel body size clines of Drosophila melanogaster. Genetics, 153, 1775-1787.

HARD, J. J., BRADSHAW, W. E. AND HOLZAPFEL, C. M. 1992. Epistasis and genetic divergence of photoperiodism between populations of the pitcher-plant mosquito, Wyeomyia smithii. Genetics, 131, 389-396.

HARSHMAN, L. G., HOFFMANN, A. A. AND ClARK, A. G. 1999. Selection for starvation resistance in Drosophila melanogaster: physiological correlates, enzyme activities and multiple stress responses. J. Evol. Biol., 12, 370-379.

HERCUS, M. J. AND HOFFMANN, A. A. 1999. Desiccation resistance in interspecific Drosophila crosses: genetic interactions and trait correlations. Genetics, 151, 1493-1502.

HOFFMANN, A. A. AND HARShMAN, L. G. 1999. Desiccation and starvation resistance in Drosophila: patterns of variation at the species, population and intrapopulation levels. Heredity, 83, 637-643.

HOFFMANN, A. A. AND PARSONS, P. A. 1989. Selection for increased desiccation resistance in Drosophila melanogaster: additive genetic control and correlated responses for other stresses. Genetics, 122, 837-845.

HOFFMANN, A. A. AND PARSONS, P. A. 1993. Direct and correlated responses to selection for desiccation resistance: a comparison of Drosophila melanogaster and D. simulans. J. Evol. Biol., 6, 643-657.

HOFFMANN, A. A., HAllas, R., SINClAIR, C., AND MITROVSKI, P. (in press). Levels of variation in stress resistance in Drosophila among strains, local populations and geographic regions: patterns for desiccation, starvation, cold resistance and associated traits. Evolution.

KARAN, D., DAHIYA, N., MUNJAL, A. K., GIBERT, P., MORETEAU, B., PARKASH, R. ET AL. 1998. Desiccation and starvation tolerance of adult Drosophila: opposite latitudinal clines in natural populations of three different species. Evolution, 53, 825-831.

KEARSEY, M. J. AND POONI, H. S. 1996. The Genetical Analysis of Quantitative Traits. Chapman \& Hall, London.

LYNCH, M. AND WALSH, B. 1998. Genetics and Analysis of Quantitative Traits. Sinauer Associates, Sunderland, MA.

MAther, K. 1966. Variability and selection. Proc. R. Soc. B, 164, 328-340.

MATHER, K. AND JinKs, J. L. 1982. Biometrical Genetics: The Study of Continuous Variation. Cambridge University Press, Cambridge.

MCKeNZIE, J. A. AND PARSONS, P. A. 1974. The genetic architecture of resistance to desiccation in populations of Drosophila melanogaster and D. simulans. Aust. J. Biol. Sci., 27, 441-456.

PARSONS, P. A. 1980. Adaptive strategies in natural populations of Drosophila. Theor. Appl. Genet., 57, 261-266. 
RIESEBERG, L. H., ARCHER, M. A. AND WAYNE, R. K. 2000. Transgressive segregation, adaptation and speciation. Heredity, 83, 363-372.

ROBINSON, S. J. W., ZWAAN, B. AND PARTRIDGE, L. 2000. Starvation resistance and adult body composition in a latitudinal cline of Drosophila melanogaster. Evolution, 54, 1819-1824.

SAS INSTITUTE. 1997. JMP statistics for Macintosh version 3.2.2. SAS Institute Inc., Cary, NC, USA.

STARMER, W. T., POLAK, M., WOLF, L. L. AND BARKER, J. S. F. 1998. Reproductive characteristics of the flower breeding Drosophila hibisci Bock (Drosophilidae) in eastern Australia: genetic and environmental determinants of ovariole number. Evolution, 52, 806-815.
WADE, M. J. AND GOODNIGHT, C. J. 1991. Wright's shifting balance theory: an experimental study. Science, 253, 10151018.

WHITLOCK, M. C., PHILIPS, P. C., MOORE, F. B. G. AND TONSOR, S. J. 1995. Multiple fitness peaks and epistasis. Ann. Rev. Ecol. Syst., 26, 601-629.

WRIGHT, s. 1931. Evolution in mendelian populations. Genetics, 16, 97-159.

WRIGHT, s. 1977. Evolution and the Genetics of Populations, vol. 3. Experimental Results and Evolutionary Deductions. University of Chicago Press, Chicago. 